\title{
Trends in health-related behaviors of Korean adults: study based on data from the 2008-2014 Community Health Surveys
}

\author{
Yang Wha Kang ${ }^{1}$, Yun Sil Ko ${ }^{1}$, Keon Yeop Kim², Changhyun Sung ${ }^{3}$, Dong Han Lee ${ }^{1}$, \\ Eunkyeong Jeong ${ }^{1}$ \\ 'Division of Chronic Disease Control, Center for Disease Prevention, Korea Centers for Disease Control and Prevention, Cheongju; \\ 2Department of Preventive Medicine, Kyungpook National University School of Medicine, Daegu; ${ }^{3}$ Division of Health Promotion, Ministry of \\ Health and Welfare, Sejong, Korea
}

\begin{abstract}
Unhealthy lifestyle behaviors such as smoking, alcohol consumption, and physical inactivity (multiple risks) often lead to serious health consequence and impaired health status. The purpose of this study was to investigate the trend in health-related behavioral factors over time among adults in South Korea (hereafter Korea). The data of 1,595,842 Koreans older than 19 years who participated in the 2008-2014 Korea Community Health Survey were analyzed to assess the trend in the prevalence of behavioral risk factors. Individual or clustering health-related behaviors were assessed according to sex, age, and region among 228,712 adults who participated in the 2014 survey. From 2008 to 2014, the age-adjusted prevalence of obesity and high-risk alcohol use increased the prevalence of male current smoking and marginally decreased walking ability. Over 7 years, the percentage of adults who reported having all three healthy behaviors (i.e., currently not smoking, not consuming alcohol or having high-risk alcohol use, and engaging in walking) decreased from $35.2 \%$ in 2008 to $29.6 \%$ in 2014. Increased efforts to emphasize multiple health-related behavioral risk factors, including reducing alcohol use and smoking, and to encourage walking are needed in the thirties and forties age groups in Korea.
\end{abstract}

KEY WORDS: Community Health Survey, Health-related behavior, Korea, Trend

\section{INTRODUCTION}

As South Korea (hereafter Korea) continues to develop economically, the average life expectancyof its population has also increased from 61.9 years in 1970 to 81.4 years in 2012. However, the current disability adjusted life expectancy is 73.0 years, showing a gap with the average life expectancy [1,2].A dispari-

\section{Correspondence: Eunkyeong Jeong}

Division of Chronic Disease Control, Center for Disease Prevention, Korea Centers for Disease Control and Prevention, 187 Osongsaengmyeong 2(i)-ro, Heungdeok-gu, Cheongju 28159, Korea

Tel: +82-43-719-7300, Fax: +82-43-719-7458, E-mail: jeong68@korea.kr

Received: Aug 12, 2015, Accepted: Sep 26, 2015, Published: Sep 29, 2015

This article is available from: http://e-epih.org/

(C) 2015, Korean Society of Epidemiology

(C) This is an open-access article distributed under the terms of the Creative Commons Attribution License (http://creativecommons.org/licenses/by/3.0/), which permits unrestricted use, distribution, and reproduction in any medium, provided the original work is properly cited. ty in community health status based on standardized mortality ratio was observed between regions [3]. Moreover, the number of patients with chronic illnesses is increasing owing to increasing population of elderly individuals older than 65 years. This has led to an increased interest in the burden of disease and health-related quality of life. The gap between the average life expectancy and disability-adjusted life expectancy, the health disparity between regions, and the trends in disease patterns can be considered as results of socioeconomic developments, changes in lifestyle, and regional differences. According to the Alameda County Study, not smoking, reduced alcohol consumption, regular physical activity, maintenance of a healthy weight, adequate amount of sleep, regular breakfast consumption, and snacking were proposed as health-related behavioral factors. Furthermore, these healthy behaviors correlated with mortality rate, either independently or in combination [4]. Healthy behaviors such as not smoking, moderate alcohol consumption, exercise, and healthy eating habits are known to prevent chron- 
ic diseases, including cancer, stroke, and cardiovascular diseases. Hence, we used the Korean Community Health Survey (CHS) to survey adults older than 19 years in Korea in order to investigate changes in main health-risk behaviors (smoking, alcohol consumption, engaging in walking, and obesity) and multiple health-related behaviors, as well as regional differences in these changes, over a period of 7 years (2008 to 2014).

\section{MATERIALS AND METHODS}

We analyzed long-term data collected through the CHS over the period 2008 to 2014. The survey population of the CHS consisted of adults older than 19 years who were living residential housing (apartments and regular houses) in tong, ban/li, areas, in 254 cities, counties, and districts throughout the country. In the CHS, sample points were determined by using tong, ban/li, as the primary sampling unit, and the households surveyed were determined by using household as the secondary sampling unit. The final study subjects consisted of approximately 230,000 individuals for each year and 254 health centers from across the country, with an average of 450 households or approximately 900 household members per health center. Each result was weighted accordingly to represent local residents and was normalized for sex and age based on the estimated population in 2005 [5].

Among the indexes calculated by using data from the CHS, smoking, alcohol consumption, walking, and obesity were used as variables in the present study. First, to investigate changes in the main health-risk behaviors in the period 2008 to 2014, we examined the changes in the medians values of 4 indexes for each city and county, namely male current smoking rate, highrisk drinking rate, walking rate, and obesity rate (self-recorded). We then classified the indexes according to their rating in a scale of 10 and presented the data visually by using the geographic information system. Male current smoking rate was defined as the fraction of men who were currently smoking and had smoked more than 5 packs (100 cigarettes) in their lifetime. High-risk drinking rate was defined as the fraction of individuals who had been drinking in the recent year and drinking one sitting, at least twice per week, 7 drinks for males and 5 drinks for females. Walking rate was defined as the fraction of individuals who walked for at least 30 minutes, 5 days per week. Obesity rate (self-recorded) was defined as the fraction of individuals who had a body mass index greater than $25 \mathrm{~kg} / \mathrm{m}^{2}$, calculated by using their known height and weight.

We examined the change in the number of three types of health-risk behaviors (not currently smoking, low-risk alcohol use, and engaging in walking) from 2008 to 2014. By using data from the 2014 CHS, we investigated the difference in multiple healthy behaviors according to sex, age, and region. Current non-smokers were defined as subjects who were not currently smoking. Low-risk alcohol drinkers were all the subjects who were not considered high-risk alcohol drinkers.

The study protocol was approved by the institutional review board (IRB) of Korea Centers for Disease Control and Prevention (IRB no. 2014-08EXP-09-4C-A).

\section{RESULTS}

\section{Change in the indexes of the major health-risk} behaviors (smoking, alcohol consumption, engaging in walking, and obesity) for each year (2008-2014)

The median male current smoking rate in each city, county, and district in the 7-year period increased marginally from $49.2 \%$ in 2008 to $50.4 \%$ in 2009 . However, it has been continuously decreasing since then, from $48.4 \%$ in 2010 to $47.1 \%$ in $2011,46.4 \%$ in $2012,45.8 \%$ in 2013 , and $45.3 \%$ in 2014 . The regional difference in male current smoking rate (maximum value - minimum value) declined from $30.8 \%$ in 2008 to $27.1 \%$ in 2012 but increased to $32.2 \%$ in 2014. The high-risk drinking rate decreased from $18.4 \%$ in 2008 to $14.9 \%$ in 2010 and then increased back to $18.7 \%$ in 2014. The regional difference in highrisk drinking rate fluctuated from $24.8 \%$ in 2008 to $21.9 \%$ in $2010,19.3 \%$ in 2013 , and then to $22.9 \%$ in 2014 . In particular, walking rate, which represents physical activity, continuously decreased from $50.6 \%$ in 2008 to $49.4 \%$ in $2009,43.0 \%$ in $2010,41.7 \%$ in $2011,40.8 \%$ in $2012,38.2 \%$ in 2013 , and $37.5 \%$ in 2014 . The regional difference in walking rate decreased from $75.7 \%$ in 2008 to $52.7 \%$ in 2014 . Obesity rate (selfrecorded) increased from $21.6 \%$ in 2008 to $22.8 \%$ in 2009 , $22.5 \%$ in $2010,23.4 \%$ in $2011,23.4 \%$ in $2012,24.1 \%$ in 2013 , and $25.3 \%$ in 2014 . The regional difference in obesity rate decreased from $20.4 \%$ in 2008 to $16.5 \%$ in 2013 but increased to $17.3 \%$ in 2014 (Figure 1).

\section{Practice rate for the main healthy behaviors (not smoking, low-risk alcohol use, and walking)}

To understand the extent local residents practiced healthy behaviors over the 7-year period, we examined the change in the three main indexes they practiced. For the indexes investigated (not currently smoking, low-risk alcohol use, and walking), 3.7\% to $4.5 \%$ of the subjects did not practice any of these behaviors. This percentage slightly increased from $2.8 \%$ in 2008 to $4.4 \%$ in 2013 and 2014. The percentage of those who practiced 1 or 2 of the behaviors showed an increasing trend every year from $60.6 \%$ in 2008 to $65.9 \%$ in 2014. Finally, the percentage of those who practiced all three indexes decreased in the past 7 years from $32.2 \%$ in 2008 to $29.6 \%$ in 2014 (Figure 2). 


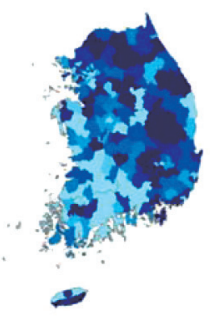

2008 Male current smoking rate

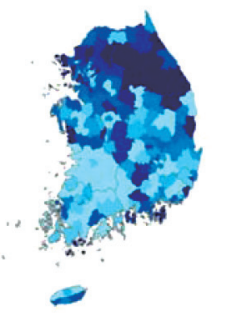

2008 High-risk drinking rate

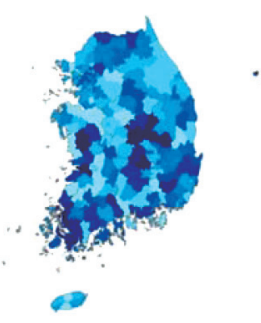

2008 Walking rate

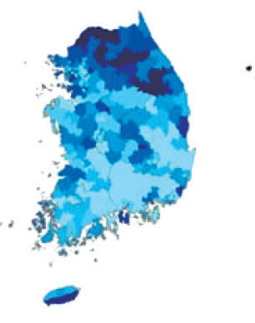

2008 Obesity rate

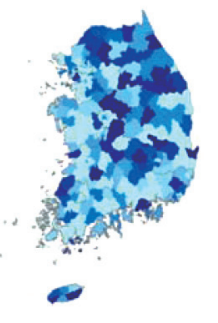

2012 Male current smoking rate

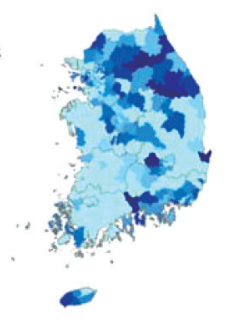

2012 High-risk drinking rate

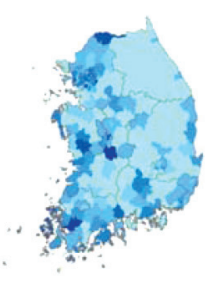

$\Leftrightarrow$

2012 Walking rate

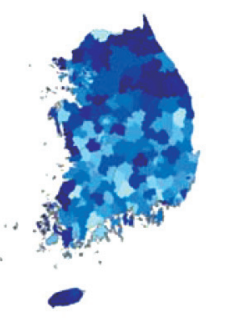

2012 Obesity rate

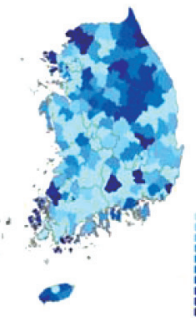

2014 Male current smoking rate

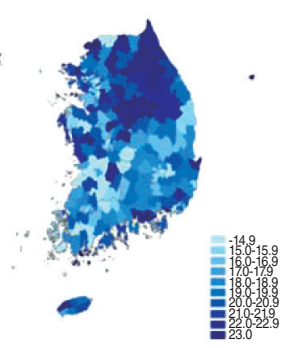

2014 High-risk drinking rate

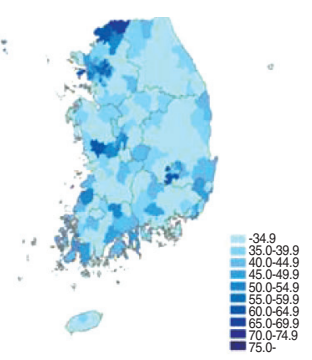

2014 Walking rate

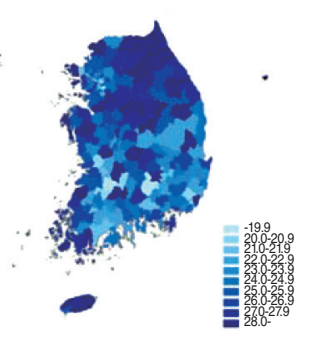

2014 Obesity rate

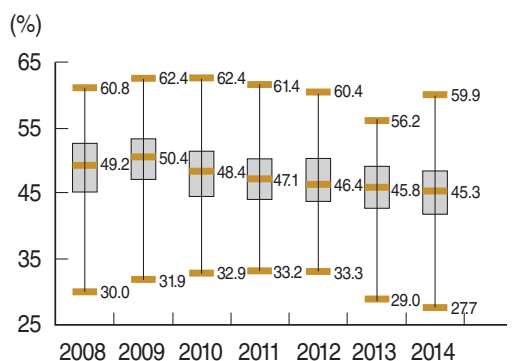

Distribution of male current smoking rates in 2008-2014, from minimum to maximum A

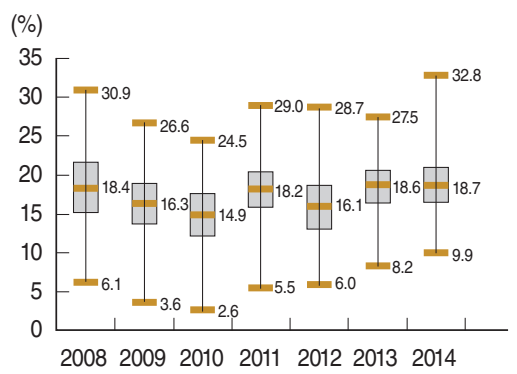

Distribution of high-risk drinking rate in 2008-2014, from minimum to maximum

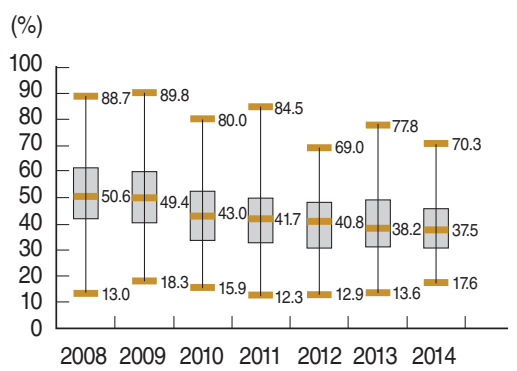

2008200920102011201220132014

Distribution of walking rate in 2008-2014, from minimum to maximum

C

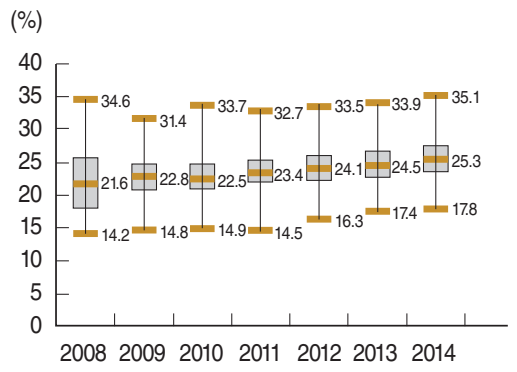

Distribution of obesity rate in 2008-2014, from minimum to maximum

Figure 1. Age-adjusted and sex-adjusted trends in the prevalence of health-related risk behaviors during the period $2008-2014$ in Korea, according to the Community Health Survey. (A) Male current smoking rate, (B) high-risk drinking rate, (C) walking rate, and (D) obesity rate.

When examined according to age, the healthy behavior practice rates were lower in the thirties and forties age groups than in the other age groups. Of the subjects, $5.6 \%$ who were in their thirties and $6.6 \%$ who were in their forties did not practice heal- 


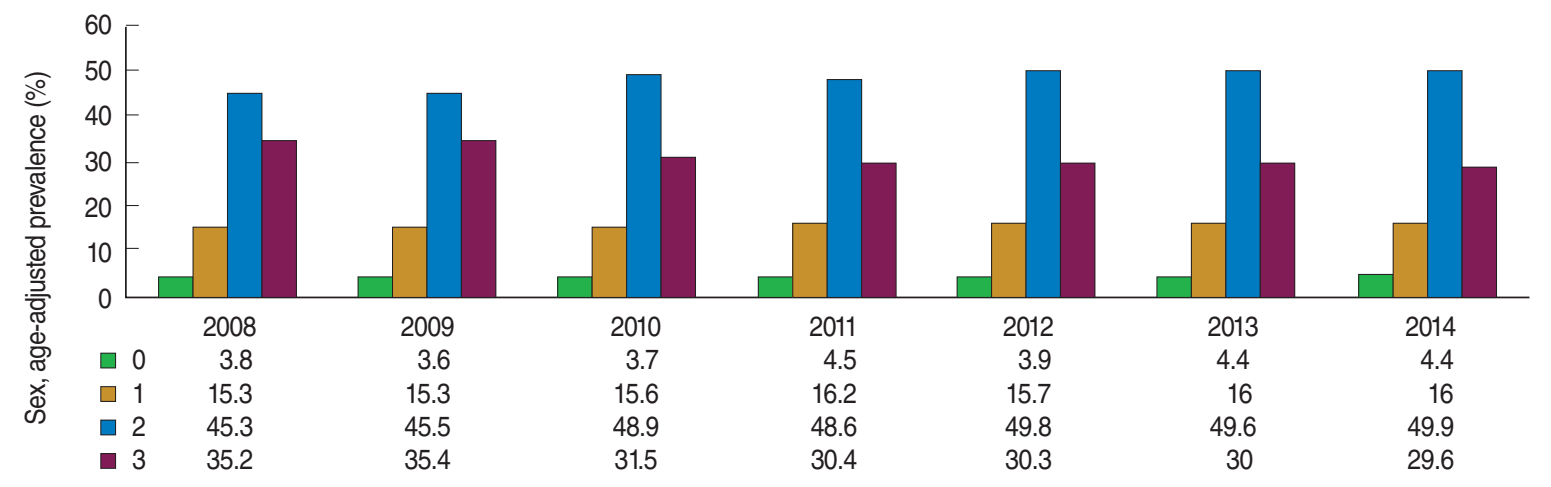

Figure 2. Sex-adjusted and age-adjusted prevalence of having 0 to 3 healthy behaviors, including not currently smoking, no alcohol consumption, no high-risk alcohol use, and engaging in walking as physical activity, during the period 2008-2014 in Korea, according to the Community Health Survey.

Table 1. Trend of the regional prevalence of health-related behaviors in 2014, according to the Community Health Survey $(n=228,721)$

\begin{tabular}{|c|c|c|c|c|c|c|c|c|c|c|c|c|}
\hline & \multicolumn{12}{|c|}{ Health-related behaviors (\%) } \\
\hline & \multicolumn{4}{|c|}{ Total } & \multicolumn{4}{|c|}{ Male } & \multicolumn{4}{|c|}{ Female } \\
\hline & $0^{1}$ & 1 & 2 & 3 & 0 & 1 & 2 & 3 & 0 & 1 & 2 & 3 \\
\hline Overall & 4.4 & 16.0 & 49.9 & 29.6 & 8.2 & 28.1 & 42.2 & 21.3 & 0.5 & 4.2 & 57.5 & 37.6 \\
\hline \multicolumn{13}{|l|}{ Age (yr) } \\
\hline $19-29$ & 3.1 & 13.8 & 47.6 & 35.4 & 5.2 & 21.9 & 43.6 & 29.2 & 0.9 & 5.2 & 51.8 & 42.0 \\
\hline $30-39$ & 5.6 & 19.4 & 51.2 & 23.8 & 10.1 & 32.8 & 41.0 & 15.9 & 0.8 & 5.3 & 61.9 & 32.0 \\
\hline $40-49$ & 6.6 & 19.7 & 48.6 & 25.0 & 12.5 & 34.4 & 38.8 & 14.3 & 0.5 & 4.5 & 58.7 & 36.1 \\
\hline $50-59$ & 4.5 & 16.9 & 48.7 & 29.8 & 8.7 & 30.3 & 42.2 & 18.7 & 0.3 & 3.4 & 55.3 & 40.9 \\
\hline $60-69$ & 1.9 & 10.6 & 50.3 & 37.2 & 3.8 & 20.5 & 46.6 & 28.9 & 0.2 & 2.0 & 53.4 & 44.3 \\
\hline$\geq 70$ & 0.5 & 6.4 & 59.3 & 33.5 & 1.4 & 13.5 & 49.4 & 35.4 & 0.0 & 2.4 & 65.0 & 32.4 \\
\hline \multicolumn{13}{|l|}{ Region } \\
\hline Seoul & 2.9 & 12.8 & 44.9 & 39.2 & 5.3 & 22.6 & 43.2 & 28.7 & 0.5 & 3.3 & 46.6 & 49.4 \\
\hline Busan & 4.6 & 16.1 & 48.8 & 30.5 & 8.8 & 28.4 & 41.1 & 21.6 & 0.4 & 4.0 & 56.3 & 39.2 \\
\hline Daegu & 3.8 & 17.1 & 49.4 & 29.6 & 7.3 & 30.8 & 41.4 & 20.4 & 0.2 & 3.6 & 57.3 & 38.6 \\
\hline Incheon & 4.0 & 16.1 & 46.2 & 33.6 & 7.2 & 27.2 & 41.5 & 24.0 & 0.9 & 5.2 & 50.8 & 43.0 \\
\hline Gwangju & 4.6 & 16.2 & 55.2 & 24.0 & 8.8 & 29.1 & 44.9 & 17.3 & 0.5 & 3.6 & 65.3 & 30.6 \\
\hline Daejeon & 3.4 & 14.9 & 47.0 & 34.5 & 6.2 & 25.7 & 41.9 & 25.9 & 0.7 & 4.3 & 51.9 & 42.9 \\
\hline Ulsan & 4.0 & 15.9 & 52.5 & 27.6 & 7.5 & 28.1 & 42.6 & 21.9 & 0.6 & 4.0 & 62.2 & 33.1 \\
\hline Sejong & 4.2 & 15.7 & 56.0 & 24.2 & 8.2 & 27.0 & 45.6 & 19.2 & 0.2 & 4.6 & 66.1 & 29.1 \\
\hline Kyonggi & 4.7 & 16.4 & 50.9 & 28.0 & 8.9 & 28.6 & 42.5 & 19.9 & 0.6 & 4.4 & 59.0 & 35.9 \\
\hline Gangwon & 6.0 & 19.2 & 52.4 & 22.3 & 11.6 & 32.0 & 39.9 & 16.5 & 0.6 & 6.5 & 64.7 & 28.1 \\
\hline Chungbuk & 5.8 & 18.8 & 52.3 & 23.1 & 11.0 & 31.6 & 41.1 & 16.3 & 0.8 & 6.3 & 63.2 & 29.7 \\
\hline Chungnam & 5.0 & 17.0 & 54.2 & 23.7 & 9.5 & 29.8 & 42.1 & 18.4 & 0.7 & 4.4 & 66.0 & 28.8 \\
\hline Jeonbuk & 4.2 & 17.1 & 54.3 & 24.4 & 8.2 & 30.2 & 43.0 & 18.5 & 0.2 & 4.2 & 65.5 & 30.1 \\
\hline Jeonnam & 4.1 & 15.8 & 50.9 & 29.1 & 8.0 & 28.6 & 41.7 & 21.5 & 0.2 & 3.3 & 59.9 & 36.4 \\
\hline Gyeongbuk & 5.7 & 17.6 & 54.5 & 22.2 & 10.9 & 31.7 & 42.5 & 14.9 & 0.6 & 3.8 & 66.3 & 29.3 \\
\hline Gyeongnam & 6.0 & 18.9 & 53.6 & 21.5 & 11.7 & 32.6 & 40.8 & 14.8 & 0.5 & 5.4 & 66.2 & 27.9 \\
\hline Jeju & 5.9 & 19.1 & 53.6 & 21.3 & 10.7 & 33.4 & 39.3 & 16.6 & 1.2 & 5.0 & 67.6 & 26.0 \\
\hline
\end{tabular}

${ }^{1}$ Having 0 to 3 healthy behaviors, including not currently smoking, no alcohol consumption, no high-risk alcohol use, and engaging in walking as physical activity.

thy behaviors. These percentages were higher than the percentages in the twenties $(3.1 \%)$, sixties $(1.9 \%)$, and "older than 70 years" age groups $(0.5 \%)$. All three healthy behaviors were practiced by $23.8 \%$ of the subjects in the thirties age group and by $25.0 \%$ of the subjects in the forties age group. These percentages were lower than that in the twenties (35.4\%), sixties
(37.2\%), and "older than 70 years" age groups (33.5\%). When differences were examined according to sex, females younger than 70 years (19 to 69 years old) were more prevalent than males of the same age range among those who practiced all three healthy behaviors. In particular, $40.9 \%$ of females in their fifties practiced all three healthy behaviors, indicating a $22.2 \%$ 
higher percentage than the percentage of males (18.7\%). Moreover, the fifties age group showed most significant difference between males and females. By region, the prevalence of those who practiced all three healthy behaviors was highest in Seoul (39.3\%), followed by Daejeon (34.5\%) and Incheon (33.6\%), and lowest in Jeju (21.3\%), followed by Gyeongnam (21.5\%) and Gyeongbuk (22.2\%), showing regional differences. Furthermore, females were more likely to practice all three healthy behaviors in all regions, except Jeju. In particular, almost 20\% more women than men living in Seoul, Busan, and Daegu practiced all three healthy behaviors, showing a large difference between sexes (Table 1).

\section{DISCUSSION}

Based on the changes in the median values of the major healthrisk behaviora lfactors according to city and county among adults in Korea, as shown in the CHS in the aforementioned 7-year period, the male current smoking rate is continuously declining. However, because of the constantly decreasing walking rate and the increasing obesity rate, an appropriate intervention is needed at both the national and local levels. Although regional differences in walking rate have been decreasing, the regional difference was as large as $52.7 \%$ (17.6\% to $70.3 \%)$ in 2014 . Regional differences in male current smoking and obesity rates had also been decreasing until recently but increased again to $32.3 \%$ and $17.3 \%$, respectively, in 2014 . Therefore, regional differences in male current smoking and obesity rates should be monitored.

In terms of the change in the practice rate of the three representative healthy behaviors (not smoking, reduced alcohol consumption, and walking) over the 7-year period, the number of individuals who practiced 1 or 2 of the healthy behaviors increased, whereas the number of those who practiced all three healthy behaviors decreased. Individuals who practiced all three healthy behaviors accounted for $29.6 \%$ of the subjects in the 2014 CHS, and those who did not practice any of the three healthy behaviors accounted for $4.4 \%$. Because the practice rate of healthy behaviors was lower in males than in females and in those in their thirties and forties than in those in their twenties and fifties, local health promotion strategies that target these age groups are needed. The practice rate for healthy behaviors in 17 metropolitan areas showed regional differences. Therefore, measures to improve health-related behaviors should be implemented at the metropolitan level for regions with low practice rates.

Healthy behaviors influence the development of cardiovascular diseases, the incidence of cancer, general mortality and morbidity, and health status. In particular, smoking is the most im- portant predictive factor of diseases and death, and has been highlighted as the priority of health objectives at the national level $[6,7]$. Excessive drinking can cause cerebrovascular diseases by increasing blood pressure [8], and obesity and lack of walking or other exercises affect morbidity and mortality, showing a close relationship to cardiovascular diseases [9-11]. In particular, for individuals who practice good healthy behaviors, the average life expectancy is 11 years longer than those who do not practice good healthy behaviors. Because $28 \%$ of males and $43 \%$ of females who ranked highly in practicing healthy behaviors showed low mortality rates, healthy behaviors were reported to affect mortality rate [12]. Ultimately, practicing healthy behaviors not only improves individual health status and prevents diseases but also contributes to health promotion among community residents. Along with the use of an individual approach that focuses on improving individual lifestyle for health promotion, a population approach that targets communities and improves local health behavioral factors should be developed in the future, both at the regional and national levels. To achieve this, CHSs must be continuously administered to provide systematic and accumulated community health data for research studies that investigate regional differences and changes in community health indexes. Studies on community health outcomes of community interventions and follow-up observations are also needed.

\section{ACKNOWLEDGEMENTS}

This research was supported by a grant from the Health Promotion Fund of Korea (090-091-4800-4849-306).

\section{CONFLICT OF INTEREST}

The authors have no conflicts of interest to declare for this study.

\section{SUPPLEMENTARY MATERIAL}

Supplementary material (Korean version) is available at http: //www.e-epih.org/.

\section{ORCID}

Yang Wha Kang http://orcid.org/0000-0002-0248-3021

Keon Yeop Kim http://orcid.org/0000-0002-1192-1767

Eunkyeong Jeong http://orcid.org/0000-0002-4386-201X 


\section{REFERENCES}

1. Korean Statistical Information Service. Cause of death statistics; 2013 [cited 2015 May10]. Available from: http://kosis.kr/statHtml/statHtml.do?orgId=101\&tblId=DT_1B34E13\&conn path=I3 (Korean).

2. Korea Health Promotion Foundation. Health Plan 2020 annual report; 2014 [cited 2015 May10]. Available from: http://www.khealth. or.kr/BoardType67.do?bid=62\&mid=361\&cmd=_view\&dept $=\& \mathrm{mnu}$ $=\& \mathrm{smnu}=\& \mathrm{idx}=8509$ (Korean).

3. Kim JH, Yoon TH. Comparisons of health inequalities in small areas with using the standardized mortality ratios in Korea. J Prev Med Public Health 2008;41:300-306 (Korean).

4. Housman J, Dorman S. The Alameda County study: a systematic, chronological review. Am J Health Educ 2005;36:302-308.

5. Korea Centers for Disease Control and Prevention. Community Health Survey [cited 2015 Aug 12]. Available from: https://chs.cdc.go.kr/ chs/ (Korean).

6. US Department of Health and Human Service. The health consequences of smoking--50 years of progress: a report of the Surgeon
General, 2014: executive. Rockville: US Department of Health and Human Services; 2014, p. 1-19.

7. Kang HY, Kim HJ, Park TK, Jee SH, Nam CM, Park HW. Economic burden of smoking in Korea. Tob Control 2003;12:37-44.

8. Rantakömi SH, Kurl S, Sivenius J, Kauhanen J, Laukkanen JA. The frequency of alcohol consumption is associated with the stroke mortality. Acta Neurol Scand 2014;130:118-124.

9. Kokkinos P. Cardiorespiratory fitness, exercise, and blood pressure. Hypertension 2014;64:1160-1164.

10. Mathieu RA 4th, Powell-Wiley TM, Ayers CR, McGuire DK, Khera A, Das SR, et al. Physical activity participation, health perceptions, and cardiovascular disease mortality in a multiethnic population: the Dallas Heart Study. Am Heart J 2012;163:1037-1040.

11. Froelicher VE. The effects of chronic exercise on the heart and on coronary atherosclerotic heart disease: a literature survey; 1976 [cited 2015 Aug 12]. Available from: http://www.dtic.mil/dtic/tr/fulltext/u2/ a022493.pdf.

12. Nandi A, Glymour MM, Subramanian SV. Association among socioeconomic status, health behaviors, and all-cause mortality in the United States. Epidemiology 2014;25:170-177. 